\title{
SRT AS A FOURTH-ORDER-THEORY WITH ANALOGY-MODEL OF DAMPED RESONANCE
}

\author{
Holger Döring \\ IQ Berlin-Spandau, \\ Germany \\ e-mail: haw-doering@t-online.de
}

\begin{abstract}
:
Discussed is an expansion of SRT to fourth-order of space-time including any velocities and possible negative kinetic energies by real rest mass in analogy of the model amplitude of enforced damped oscillation.

This paper serves an introduction to developed SRT including superluminar translating velocities without tachyons or imaginary restmass. Focused is on those parts, which develop the elaborated lorentz-transformations. Discussed are the problems of this theory.
\end{abstract}

Keywords: SRT, fourth-order-theory, any velocity, FTL, ,real rest mass,, resonance analogy, damped space-time.SR-Extrapolation, superluminal-velocity, negative kinetic energy, fourth-order worldline-element,no-tachyons

\section{Introduction:}

Local spacetime as analogy for damped oscillation-amplitude:

The well-known Lorentzian k-factor of SRT:

$$
\mathrm{k}^{+}=\sqrt{1-\frac{v^{2}}{c^{2}}} \quad ; \text { resp. } \mathrm{v}<\mathrm{c}
$$

can be interpreted as the half of an amplitude-term in analogy of undamped oscillation. Together with Feinbergs term for tachyons[2.]:

$$
\mathrm{k}^{-}=\sqrt{\frac{v^{2}}{c^{2}}-1} \text { and } \mathrm{v}>\mathrm{c}
$$

one get the whole desumption of an analogy-amplitude of an undamped oscillation curve. (Figure1). 


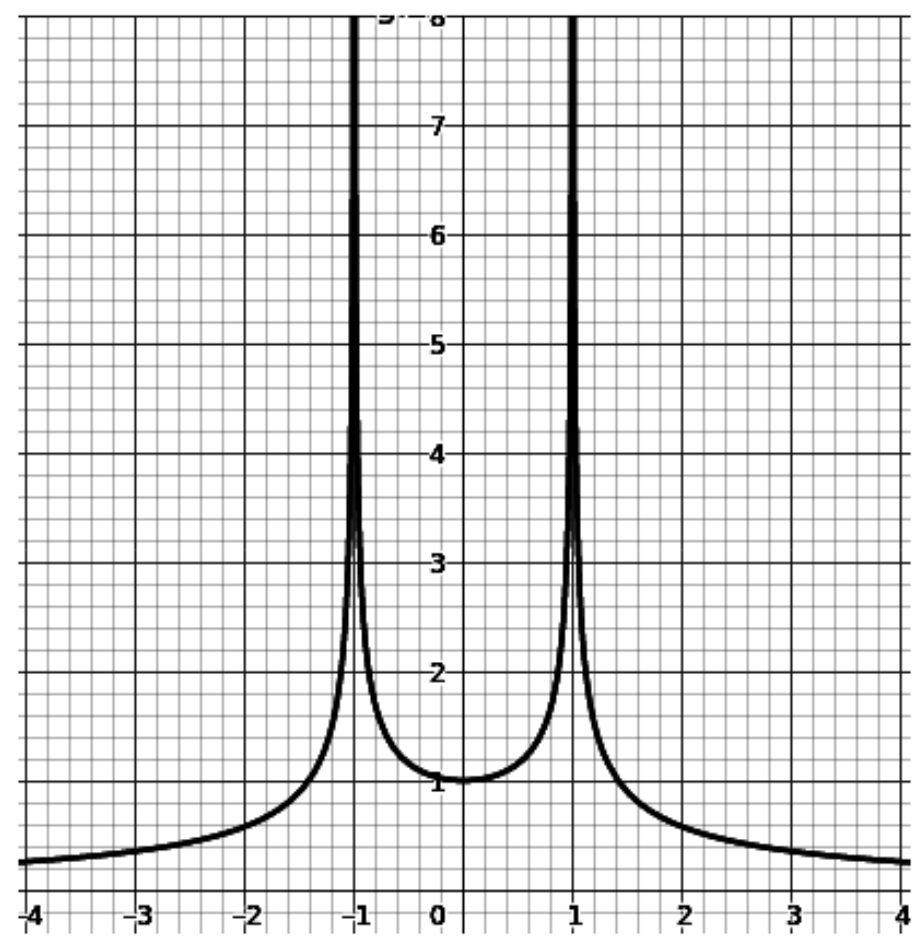

Figure 1. Shown is the analogy of amplitude of classical SRT for both $v<_{c}$ and $v>_{C}$ with infinite singularity at $v=c$ with $c=1$.

This classical model is now expanded to enforced damped oscillation.

So we get in analogy to amplitude of classical oscillation a new k-factor of:

$$
K=\sqrt[4]{\left(1-\frac{v^{2}}{c^{2}}\right)^{2}+\frac{n a^{2} v^{2}}{c^{4}}}
$$

where $n$ is a positive real number, $n \in \mathbb{R}$ and a is an extra velocity, which role has to be discussed. Suggested is a form of rotation- or oscillation velocity of the moving object (or of the local space-time) in addition to translation velocity v. (Another form of analogy is the adding of an ohm-resistor-term to a superconducted oscillation-circuit in series with coil and 
capacitor.Therefore in the model image classical SRT is „,superconducted“ and gets now a „resistorterm." But in physical principle, the both analogies are similar.)

Figures 2. shows graphs of the amplitudes of a damped harmonic oscillator as a function of the velocity of the periodic forcedriving it. Each of the curves on the graphs represents a different amount of damping. All curves peak at the point where the velocity of the driving force equals the natural velocity c of the harmonic oscillator. The highest peak, or greatest response, is for the least amount of damping, because less energy is removed by the damping

force. Note that since the amplitude grows as the damping decreases, taking this to the limit where there is no damping $(a \equiv 0)$, the amplitude becomes infinite which leads to classical SRT, with $v_{\max }=c$ which represents Figure 1. above. Figure 2.. shows the overlapping of different damped states for low, middle and strong damping analogies.

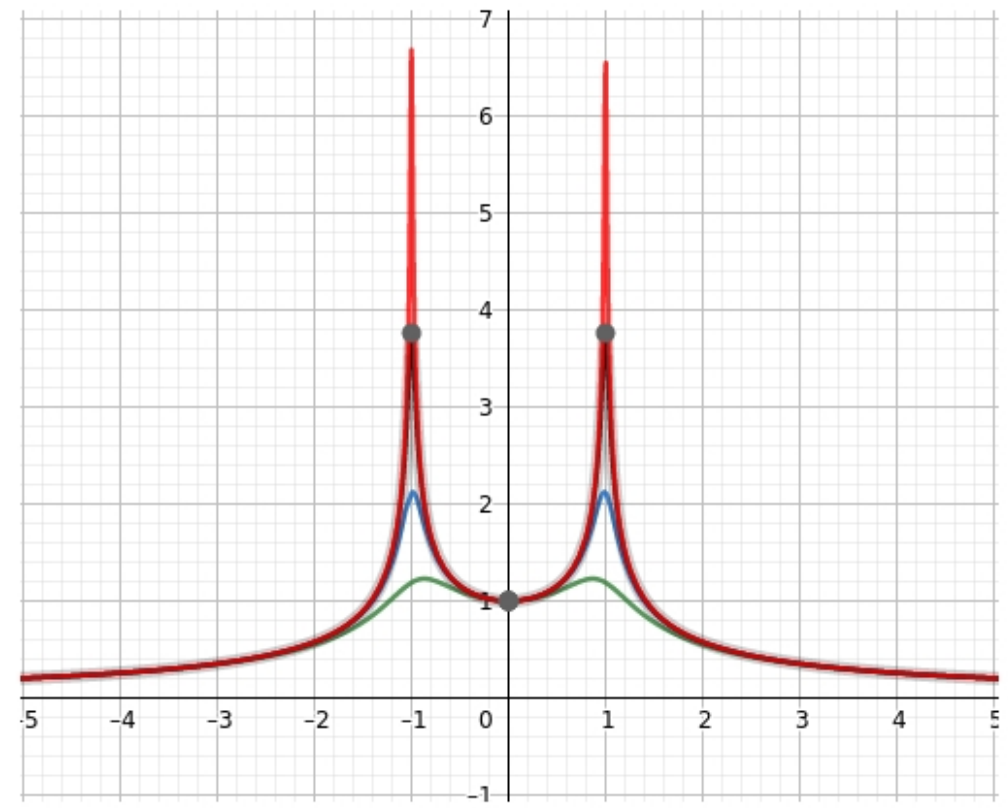

Figure 2: Shown are the damping states of modified lorenztian SRT-Kamplitude for four different values:

red: $a^{2}=0.0005 /$ black: $a^{2}=0,005 /$ blue $a^{2}=0,05 /$ green $a^{2}=0,5$. All $n=1, c=1$ 


\section{Spacetime-conditions:}

2.1. If a line-element of the new tangent-spacetime of fourth-order in two dimensions $\mathrm{x}$ and $\mathrm{t}$ is formed, there is:

$$
\left(c^{2} t^{2}-x^{2}\right)^{2}+r^{2} x^{2}=d s^{4}
$$

where $\mathrm{r}$ is a distance respectively to $\mathrm{a}=\frac{r}{t} \quad$.( $\mathrm{r}=$ const. $)$

$r$ may be a rotation radius of translating object or an oscillation wavelength $r=\lambda$ of the moving body.This would mean, that special addition velocities would not be added to k-factor in a normal, conventional way like:

$$
\sqrt{1-\frac{v^{2}+a^{2}}{c^{2}}} \quad \text { or } \quad \sqrt{1-\frac{(v+a)^{2}}{c^{2}}}
$$

but like in (3.).

\subsection{Geometry-aspects:}

In fact, the new line-element of local space time looks like a special form of one-parametring pseudo-Kummerplane (with positive sign at $\mathrm{r}^{2}$, original Kummerplanes have a negative sign). The pseudo-Kummer surfaces are a family of quartic surfaces given by the algebraic equation $\left(x^{2}+y^{2}+z^{2}-k^{2}\right)^{2}+\lambda=w$ with several bounding conditions for $\lambda$. Here seem to are no other bounding conditions than $\lambda=r$.

2.3. If the new K-term is developed into a series, one gets:

$$
\sqrt[-4]{\left(1-\frac{v^{2}}{c^{2}}\right)^{2}+\frac{n a^{2} v^{2}}{c^{4}}}=1+\frac{1}{2} \frac{v^{2}}{c^{2}} \quad\left(1-\frac{1 n a^{2}}{2 c^{2}}\right)+\frac{1 v^{4}}{4 c^{4}}+\mathrm{O}(\mathrm{n})
$$

which leads the first term of rest energy $E=m c^{2}$ unchanged, but the velocity-dependend terms of kinetic energy will be moderatly changed dependend on a in contradiction to classical SRT as 


$$
\mathrm{E}(\mathrm{kin})=\frac{1 m v^{2}}{2} \quad\left(1-\frac{1 n a^{2}}{2 c^{2}}\right)
$$

2.4. Experimental evidence:

This term could be proofed experimentally in example by spin-stabilised space-crafts because the a-term is dependend of velocity v. No such effect has been found yet, depending on rotation.[6.], [7.].We see, that the classical, non relativistic term of kinetc energy would be modified but not the rest energy term $\mathrm{E}=\mathrm{mc}^{2}$. This rest-term hat the same value as in classical SRT.

Of course, the measuring effects will be very small.

table 1:

Examples given for classical SRT k-factor (1.) and two elaborated factors $\mathrm{K}$ (3.) for technical possible, experimental low damping (velocity units v,a with $c=1$ ):

\begin{tabular}{|c|c|c|}
\hline $\mathbf{v}$ & $\mathbf{a}$ & $\mathbf{K}$ \\
\hline 0.1 & $0 \quad$ (SRT) & 0.9949874371 \\
\hline 0.1 & $10^{-4}$ & 0.9949874371 \\
\hline 0.1 & $10^{-3}$ & 0.9949874396 \\
\hline 0.5 & (SRT) & 0.8660254038 \\
\hline 0.5 & $10^{-4}$ & 0.8660254063 \\
\hline 0.5 & $10^{-3}$ & 0.8660256538 \\
\hline 0.9 & (SRT) & 0.4358898944 \\
\hline 0.9 & $10^{-4}$ & 0.4358899188 \\
\hline 0.9 & $10^{-3}$ & 0.4358923394 \\
\hline 1 & o $\quad$ (SRT) & $\mathbf{0}$ \\
\hline 1 & $10^{-4}$ & 0.01 \\
\hline 1 & $10^{-3}$ & 0.0316227766 \\
\hline
\end{tabular}

May be experimental evidence would be difficult but the testing could go over relativistic dopplereffect of rotating/oscillating bodies.

\subsection{Spacetime-transformations:}

Therefore there are the affilated Lorentz-Transformations in $\mathrm{x}$-t-direction in 2 dimensions (with $\mathrm{y}^{\mathfrak{s}}=\mathrm{y} ; \mathrm{z}^{\mathfrak{}}=\mathrm{z}$, parallel movement along the $\mathrm{x}$-axis) to construct from the transformation-matrix, wich is:

$$
\mathbf{A}=\left(\begin{array}{llll}
\boldsymbol{B} & \boldsymbol{0} & \mathbf{0} & \boldsymbol{C} \\
\mathbf{0} & \boldsymbol{E} & \mathbf{0} & \mathbf{0} \\
\mathbf{0} & \mathbf{0} & \boldsymbol{E} & \mathbf{0} \\
\boldsymbol{C} & \mathbf{0} & \mathbf{0} & \boldsymbol{B}
\end{array}\right)
$$

where the description is as follows (B,C,E,0 are 2x2 matrices): 


$$
\mathbf{B}=\left(\begin{array}{cc}
K & 0 \\
0 & K
\end{array}\right) \quad ; \quad \mathbf{C}=\left(\begin{array}{cc}
K \beta & 0 \\
0 & K \beta
\end{array}\right) \quad ; \mathbf{E}=\left(\begin{array}{ll}
1 & 0 \\
0 & 1
\end{array}\right) \quad ; \mathbf{0}=\left(\begin{array}{ll}
0 & 0 \\
0 & 0
\end{array}\right)
$$

$$
\text { with } \mathrm{K}(3 .) \quad \text { and } \quad \beta=\frac{2 v^{2}}{c^{2}}+\frac{v^{4}}{c^{4}}+\frac{n a^{2} v^{2}}{c^{4}}
$$

There is a description of two-part values like vectors with two coordinates. To get the right K-Factor of fourth order into the ansatz for the coordinates of LT's, one has to consider the conditions of coordinates as a 2- component size. Maybe interpreted as a vector, string or spinor.This may be the coupling of the two space-times of second form in line-element.

Therefore the LTs in two dimensions come from:

$$
\left.\left(\begin{array}{c}
\left(\begin{array}{c}
x^{\prime} \\
{ }_{1} \\
0
\end{array}\right) \\
\left(\begin{array}{c}
y^{\prime}{ }_{1} \\
0
\end{array}\right) \\
\left(\begin{array}{c}
z^{\prime}{ }_{1} \\
0
\end{array}\right) \\
\left(\begin{array}{c}
0 \\
0 \\
c t^{\prime}
\end{array}\right)
\end{array}\right)=\left(\begin{array}{l}
\left(x_{1}\right. \\
0
\end{array}\right)\right)\left(\begin{array}{c}
\left(\begin{array}{c}
y_{1} \\
0
\end{array}\right) \\
\left(z_{1}\right. \\
0
\end{array}\right) \cdot\left(\begin{array}{cccc}
B & 0 & 0 & C \\
0 & E & 0 & 0 \\
0 & 0 & E & 0 \\
C & 0 & 0 & B
\end{array}\right)
$$

There follow the LTs:

$$
\left(\begin{array}{c}
x^{\prime}{ }_{1} \\
0
\end{array}\right)=\mathrm{K}\left(\begin{array}{c}
x_{1}+c t_{1} \beta \\
0
\end{array}\right) \text { and }\left(\begin{array}{c}
0 \\
c t^{\prime}{ }_{1}
\end{array}\right)=\mathrm{K}\left(\begin{array}{c}
0 \\
x_{1} \beta+c t_{1}
\end{array}\right)
$$

and $\quad y^{\prime}{ }_{1}=y_{1} \quad ; \quad z^{\prime}{ }_{1}=z_{1} \quad$ and $\mathrm{K}$ (3.)

Note: for zeros in the elementary coordinate-vectors there can be second space-time-coordinates filled in for whole description.

2.6. For time-variation and length changing there is $\left(\mathrm{t}^{\mathrm{s}}, \mathrm{x}^{6}\right.$-the moving inertial system):

$$
\mathrm{t}^{\varsigma}=\frac{t}{\sqrt[4]{\left(1-\frac{v^{2}}{c^{2}}\right)^{2}+\frac{n a^{2} v^{2}}{c^{4}}}} \quad \text { and } \quad \mathrm{x}^{\varsigma}=x \cdot \sqrt[4]{\left(1-\frac{v^{2}}{c^{2}}\right)^{2}+\frac{n a^{2} v^{2}}{c^{4}}}
$$

There is also a mass variation formula: 


$$
\mathrm{m}=\frac{m_{0}}{\sqrt[4]{\left(1-\frac{v^{2}}{c^{2}}\right)^{2}+\frac{n a^{2} v^{2}}{c^{4}}}}
$$

where the kinetic mass is not alone increasing (and not into infinity) but also decreasing.This size depends of the damping force-factor a.All formulas lead for $a \equiv 0$ into classical SRT (and Feinbergs Tachyon-case).

2.7. Special case: for $\mathrm{v}=\mathrm{c}$ we get no singularity like in classical SRT but:

$$
\mathrm{m}=\frac{m_{0}}{\sqrt{\frac{\sqrt{n} a}{c}}} \quad ; \quad a \neq 0
$$

For $\mathrm{n}=1$ and $\mathrm{a}=\mathrm{c}$ this will be restmass $m=m_{0}$. But also $n=2 \pi$ can be chosen if rotation is assumed. (But this term can be included in a).

From this formulas is seen: there is also not only time-dilatation but contraction; also both for length-changing and mass variation.No singularity by $\mathrm{v}=\mathrm{c}$ and there will be no light-barrier any more.

2.8. The addition-theorem of velocities in two dimensions $\mathrm{x}, \mathrm{ct}$ will form to:

$$
\begin{gathered}
\mathrm{u}^{\mathrm{s}}=\frac{u+c \beta}{1+\frac{u \beta}{c}} \\
\text { with } \mathrm{u}^{\mathrm{s}}=\frac{\left.\| \begin{array}{c}
x^{\prime}{ }_{1} \\
0
\end{array}\right) \|}{\|\left(\begin{array}{c}
0 \\
t^{\prime}
\end{array}{ }_{1} \|\right.} \quad \text { and } \mathrm{u}=\frac{\left\|\left(\begin{array}{c}
x_{1} \\
0
\end{array}\right)\right\|}{\left\|\left(\begin{array}{c}
0 \\
t_{1}
\end{array}\right)\right\|} \\
\text { where } \beta=\frac{2 v^{2}}{c^{2}}+\frac{v^{4}}{c^{4}}+\frac{n a^{2} v^{2}}{c^{4}}
\end{gathered}
$$

2.9. There are no zero points of line element (which is seen easily in (4.)) but more accurately: By developing to $\mathrm{x}$ there is:

with $\mathrm{y}=\mathrm{ct} ; \mathrm{r}=a \cdot t \quad$ const.; $n \in \mathbb{R}$

$$
x_{1,2,3,4}= \pm \sqrt{\frac{2 y^{2}-n r^{2}}{2} \pm \sqrt{\frac{\left(2 y^{2}-n r^{2}\right)^{2}}{4}-y^{4}}} \quad \text { Mostly set } \mathrm{n}=1 \text { here. }
$$

The inner root is solved in $\mathbb{R}$ for $n r^{2} \geqslant 4 y^{2}$ whereby the outer root is only solveable in $n r^{2} \leqslant 2 y^{2}$.The contradiction is seen.Therefore there are no zero points of line element.(There are 
no light beams at the center of the coordinate-system in the event, it's dark there).The limiting case shows for $r=0$ that $x=c t$.There classical SRT line-element is back.

2.10. Some line-element curves with different a are plotted. Seen is, that the curve in analogy to amplitude of damped oscillation is steady with no singularity at $\mathrm{v}=\mathrm{c}$.Also there is the classical, relativistical real rest mass.Note that there is no statical line element like in classical SRT but the form of the line element depends on the size of $\mathrm{r}$ (which may be choosen as constant).So there is a dynamical line-element.If $r$ will be varied, the line-element of local tangent-spacetime varies either.

Instead of a static light-cone (Figure 3.,lineelement 1) there are the following graphs:(Figures 4.6.,linelements 2-4), dynamic of the examples depends on a-value resp. on given r.:

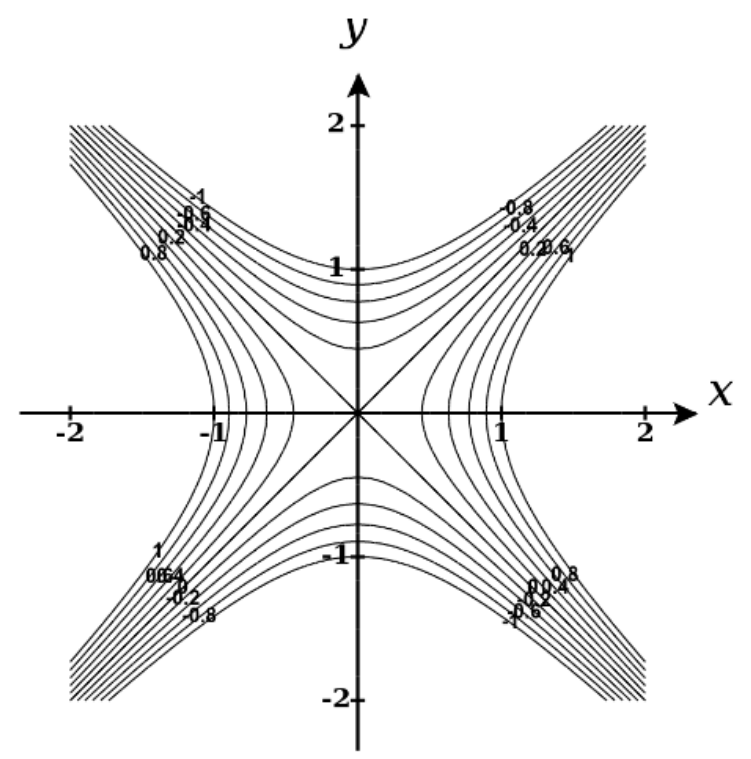

Figure 3.The picture shows classical SRT-Lightcone-element in two dimensions (rectangular coordinates chosen) with $y=c t$ and damping factor $r=0, c=1$

$$
\text { and } z=x^{2}-y^{2} ; y=c t
$$




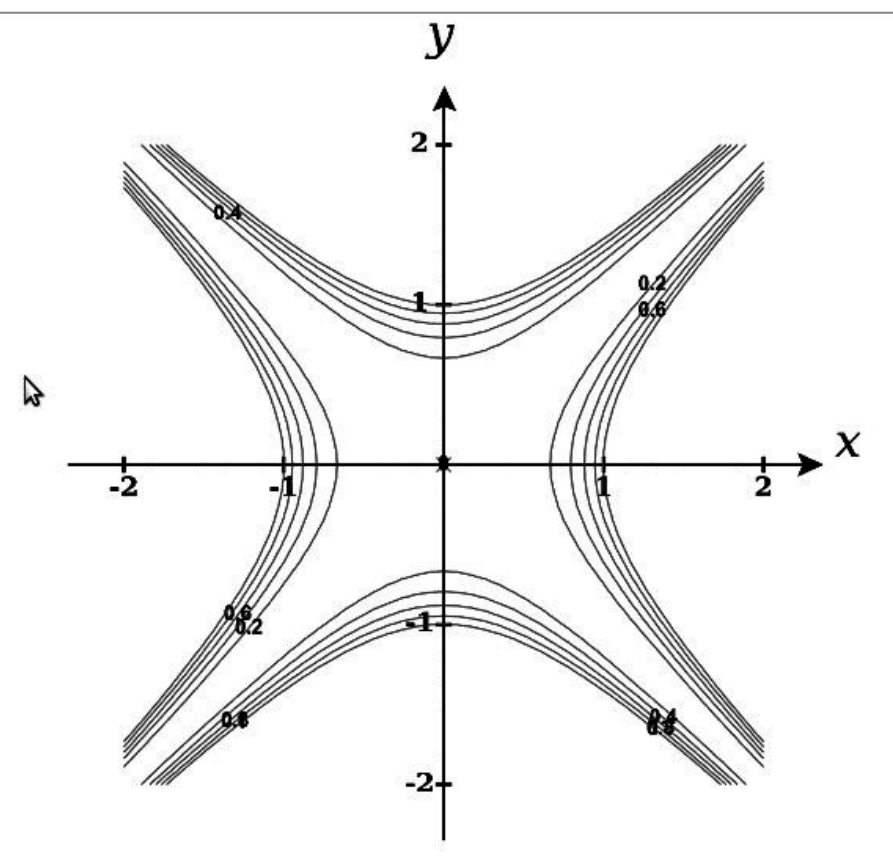

Figure 4. The picture shows a line-element with a small damping-factor $r$ of $r=0,000001$.

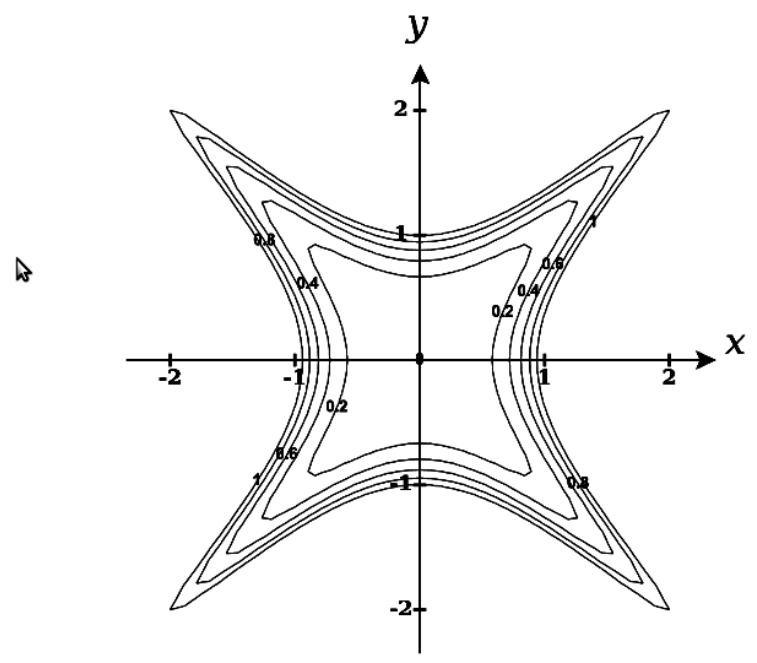

Figure 5.The picture shows a line-element with a medium damping-factor $r$ of $r=0,5$. 


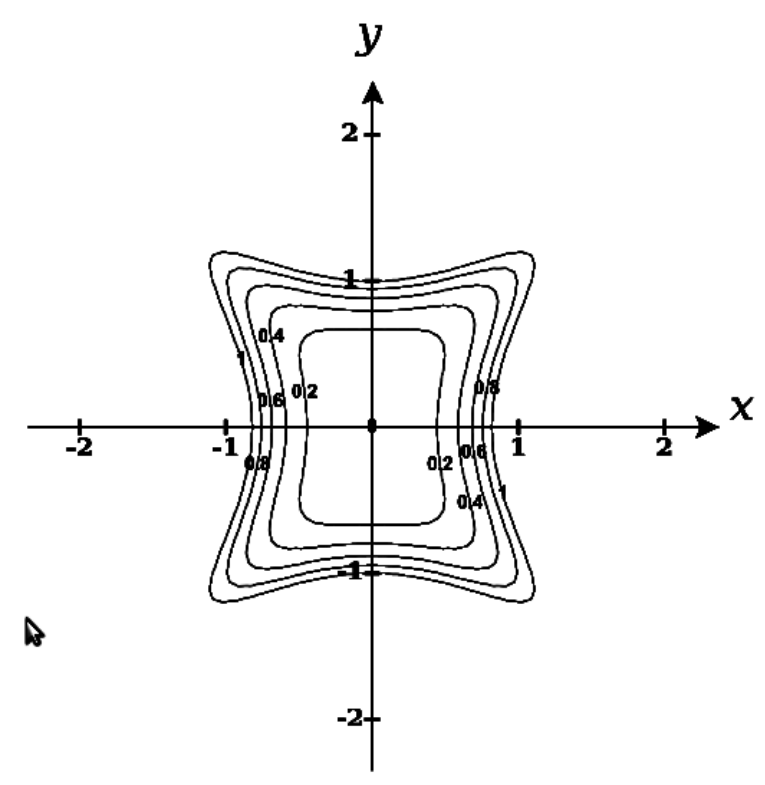

Figure 6. The picture shows a line-element with a strong damping factor $r$ of $r=0,9$.

For all graphs (4.-6.) there is the line-element $\mathrm{ds}^{4}$-form in:

$$
d s^{4}=z=x^{4}-x^{2}\left(2 y^{2}-r^{2}\right)+y^{4} ; y=c t
$$

\section{Remarks:}

The invariance-velocity c will not be any longer interpreted as a maximum speed, but only as a form of „eigenresonance“-velocity of local tangent spacetime concerning coupling of rotating/oscillating movement of material matter with translation-moving.There will be no imaginary rest mass but only the well-known conventional real rest mass of matter particles with any velocities. This may be restricted by the cut-off of negative kinetic energy..

Of course this is a dual theory. Because it can be developed in two ways.

1.The „weak-damping-analogy“model,which is near to classical SRT-model and holds the converge- condition:

$$
\frac{n a^{2} v^{2}}{c^{4}}<\left(1-\frac{v^{2}}{c^{2}}\right)^{2} \quad \text { and } \quad v \neq c \quad, \quad a=0 \text { possible. }
$$

The developing is in first order:

$$
\sqrt[4]{\left(1-\frac{v^{2}}{c^{2}}\right)^{2}+\frac{n a^{2} v^{2}}{c^{4}}}=\left(1-\frac{v^{2}}{c^{2}}\right)^{\frac{-1}{2}}-\frac{n a^{2} v^{2}}{4\left(c^{2}-v^{2}\right)^{2}}+\mathrm{O}(\mathrm{n})
$$


2.The „strong-damping-analogy“ model with the reverse condition:

$$
\frac{n a^{2} v^{2}}{c^{4}}>\left(1-\frac{v^{2}}{c^{2}}\right)^{2} \quad \text { and } \quad a, v \neq 0
$$

In first order there is this time:

$$
\sqrt[4]{\left(1-\frac{v^{2}}{c^{2}}\right)^{2}+\frac{n a^{2} v^{2}}{c^{4}}}=\frac{c}{\sqrt{n a v}}-\frac{c\left(c^{2}-v^{2}\right)^{2}}{4(n a v)^{2,5}}+\mathrm{O}(\mathrm{n})
$$

\section{Problems:}

4.1.: Of course, causality problems may occur, because we have space-time coupled coordinates in the line-element which may break isotropy conditions.

4.2.: After a certain value of a there is whole energy decreasing under restmass-energy, but positive with $\left(\lim _{a \rightarrow \infty} K\right)=0$ which means negative kinetic energies, which causes some problems in interpretation.In addition with dirac-equation, where negative energy-solutions are interpreted as charge-changing with positive energy this problem may be solved or explained.This interpretation is known though for seperated, isolated particles not for whole solid bodies. On the other hand diracequation has to be changed either, because its a relativistic equation.This phenomenon here could be interpreted as a spontan charge turn-over of the whole moved matter, when the special term of $\mathrm{v}$ with constant a is arrived in motion.

4.3.: About the constraint-Force.We do not know, what the cause of the outer force could be.Is it a low form of gravity?Is ist only „rotation/oscillation-force“ of the moving object? Is it a form of „oscillating local space-time“? (But how could this be without description of gravity, gravity-waves and curvature?) Is it a form of Lense-Thirring-effect (rotation of the local frames)?Is it a real assumption or only an analogy? Is it nothing?

4.4.:If a has something to do with ,rotation“, particle- spin has to be included without contradictions.

4.5.:If the analogy is taken seriously, there has to be some sort of „wave-equation“.

4.6.May be, this all is only a conformal transformation, because if it is set:

$$
\begin{aligned}
& \sqrt[4]{1-\aleph^{4}}=\sqrt{1-\varepsilon^{2}}, \quad \beta^{4}=\frac{2 v^{2}}{c^{2}}-\frac{v^{4}}{c^{4}}-\frac{n v^{2} a^{2}}{c^{4}} \text { there is: } \\
& \varepsilon_{1,2,3,4}= \pm \sqrt{1 \pm \sqrt{1-\aleph^{4}}} \text { with } \varepsilon=\frac{v^{\Theta 2}}{c^{\Theta 2}} \text { and there is a classical lorentzian-space in other velocity } \\
& \text { defined coordinates again. }
\end{aligned}
$$

\section{Discussion}

In analogy of amplitude of damped oscillation there is a construction of a local tangential spacetime as a „developed“ SRT with lineelement of fourth order. (This could lead to a manifold of Finsler-type). 
This theory can be interpreted as a form of unified, unbroken symmetry, which leads in case of $a \equiv 0$ to limiting different and separated SRT-states of Einstein and Feinberg in local tangentspaces.For this spacetime here movement of matter with $\mathrm{v}>\mathrm{c}$ is allowed without imaginary restmass as assumed in [3].

Also $\mathrm{v}=\mathrm{C}$ is authorized for matter with restmass $\mathrm{m}_{0}>0$. On the other hand $E_{G}=E_{0}$ occurs for $K=1$ and $v=\sqrt{2-n a^{2}}$, this means for $a \in[0 ; \sqrt{2}]$, when $c=1, n=1$.

Negative kinetic energy:

There is:

$E_{\text {kin }}=m_{0} c^{2} \cdot\left(\sqrt[4]{\left(1-\frac{v^{2}}{c^{2}}\right)^{2}+\frac{n a^{2} v^{2}}{c^{4}}}-1\right)$ with $n=1$ chosen. Therefore negative kinetic energy occurs, when whole energy decreases under restenergy.

\section{Conclusion:}

A local spacetime of fourth order as expansion of classical SRT can be constructed without a maximal speed at $\mathrm{v}=\mathrm{c}$ but there may be several problems like causality differences, negative kinetic energies or possible sponanteinous charge changing with a function of velocity which are yet not fully clear explained without contradictions.In conclusion imaginary restmasses are avoided and the existence of tachyons, which only exists as a theoretical limit-solution for the broken symmetry state of two fourspaces with different Lorentz-factors.

But there is negative kinetic energy and also causality problems on this special space-time because of multplied mixed space- and time-coordinates which may break isotropy.

\section{Appendix A:}

Instead of having a K-term like (3.):

$$
K=\sqrt[4]{\left(1-\frac{v^{2}}{c^{2}}\right)^{2}+\frac{n a^{2} v^{2}}{c^{4}}}
$$

where the additing term depends on $\mathrm{v}$ and which causes no rest-effect but only a velocity-dependent effect it can be assumed a changed term like:

$$
K=\sqrt[4]{\left(1-\frac{v^{2}}{c^{2}}\right)^{2}+\frac{n a^{4}}{c^{4}}}
$$

where experimental effects would appear at $\mathrm{v}=0$, which could be proven as a rest-measurement on earth-surface, as example for rotating or oscillating objects[4.],[5.].No such effect was found yet until today. 
Therefore is:

$$
K(v=0)=\sqrt[4]{1+\frac{n a^{4}}{c^{4}}}
$$

with a as a rotation or oscillation velocity when $v=0$.If such an effect would exist, it could be measured experimentally on surface of earth without using a space-probe.

Therefore the line-element of local tangent space-time in two dimensions will read to:

$$
\left(c^{2} t^{2}-x^{2}\right)^{2}+r^{4}=d s^{4}
$$

where $r$ is the above mentioned special distance, may be a radius $r$ or a wavelength $\lambda$ (or $r_{\text {Planck). }}$ ) A LT and other formulas for this case can also be developed easyly.

Also we can see this whole elaboration of SR-Theory as a description of vectors of planecoordinates with

$$
\begin{aligned}
x^{u v} \cdot x^{u v} \cdot \eta_{\mu v} & =d s^{4} ; \text { where } \mathrm{x}=\left(\mathrm{x}^{2}, \mathrm{y}^{2}, \mathrm{z}^{2}, \mathrm{C}^{2} \mathrm{t}^{2}\right) ; \mu v=\{0 ; 1 ; 2 ; 3\} \\
\text { and } \quad & \eta_{\mu v}=\left(\begin{array}{cccc}
1 & 1 & 1 & -1 \\
1 & 1 & 1 & -1 \\
1 & 1 & 1 & -1 \\
-1 & -1 & -1 & 1
\end{array}\right)
\end{aligned}
$$

Space-time mixed states of the local tangent-space fundamental-tensor are negative."Pure“ states are positive.

If also a Gedanken-Experiment is assumed with rotating circle-disk like in [1.],suggested by Einstein to improve curvature in SRT by measuring lenght of the circumference and diameter (radius) of the disk by unit-scales to get length-contradiction, there are news. In classical SRT there is only $U / r<2 \pi$.In this case here, there is $U / r \geq 2 \pi$ either because there is a form of length dilatation.This model may have influence on modified GRT-gravity-fields.

\section{Summary}

Well known is the Lorentz-Einstein factor of SRT:

$$
\mathrm{k}^{+}=\sqrt{1-\frac{v^{2}}{c^{2}}}
$$

This factor is interpreted as an analogy of an amplitud-factor of undamped oscillation in oscillation-theory and develop this factor to the analogy of damped state.

Then we get in full analogy:

$$
\mathrm{K}=\sqrt[4]{\left(1-\frac{v^{2}}{c^{2}}\right)^{2}+\frac{n a^{2} v^{2}}{c^{4}}}
$$


where a is a new term of damping-factor. It has the form of a velocity, which leads for $\mathrm{a}=0$ to the classical, seperated, two states of Einsteinian and Feinbergs-SRT-factors for subluminal and superluminal velocity.[1],[2],[3]

From this K-factor we now develop the affiliated tangent space-time.

The K-Factor leads to a wordline-element of fourth-order:

$$
\left(c^{2} t^{2}-R^{2}\right)^{2}+R^{2} r^{2}=d s^{4}
$$

where $\mathrm{R}^{2}=\mathrm{x}^{2}+\mathrm{y}^{2}+\mathrm{z}^{2}$ and $\mathrm{r}$ the new defined length for the „damping state“.Its origin is velocity a.

What $\mathrm{r}$ exactly physically means, has to be discussed seperately. May be it can defined as an oscillation wavelength of the object moving with velocity $\mathrm{v}$ or as a rotation radius of a rotating object. Maybe it is the eigenlength of an oscillating spacetime (like the Planck-Length). May be it is a metron-length.

If is thought of $r=$ const, similar to the characteristic of $c$, there can derived the affiliated spacetime LT transformations.

With Laplace developing-theorem there is constructed the transformation-matrix for two (or four) dimensions.Including this there is to defined the space-time coordinates as twodimensional vectorcoordinates.So there is

$\left(\mathrm{x}^{\prime}, 0\right),\left(\mathrm{ct}^{\prime}, 0\right)$ and $(\mathrm{x}, 0)$ resp. $(\mathrm{ct}, 0)$ or $\left(\mathrm{y}^{\varsigma}, 0\right),\left(\mathrm{z}^{\prime}, 0\right)$ and $(\mathrm{y}, 0),(\mathrm{z}, 0)$. Since from $\mathrm{K}$ one gets Det $\mathrm{A}=1$.This condition shows the transformation-matrix

with $K$ defined from $K=\sqrt[4]{1-b^{4}} \quad$ there is $b^{4}=\left(2 v^{2} / c^{2}-v^{4} / c^{4}-a^{4} / c^{4}\right)$

Therefore there are constructed the lorentzsimiliar transformations for the space-time in two dimensions.

In analogy of amplitude of damped oscillation there is a local tangential space-time as a „developed“ SRT with lineelement of fourth order. This could lead in GRT to a manifold of Finslertype.

But there are some problems in the matter:

First problem:

Curiosity:for $\mathrm{a}=\mathrm{c}$ and $\mathrm{v}=\mathrm{c}$, there are $\mathrm{x}^{\mathrm{s}}=\mathrm{x}$ and $\mathrm{ct}{ }^{\mathrm{s}}=\mathrm{ct}$, these are rest-coordinates.Contradiction of movement with doubleart translating and rotating velocities equal to $\mathrm{c}$ and getting restsizes of coordinates seems rather strangely. (Its like the red queen-phenomenon in „through the looking glass“, who had to run the fastest she can, to hold her place) .So this phenomenon should be called the „red-queen-phenomenon“.

Then there is the problem of negative kinetic energy. When by constant a, the objects moving with velocity $\mathrm{v}$ increases over $\mathrm{c}$ and in limes against infinity (no newtonian states) total energy rans under the limit of restmass-energy by $\mathrm{v}=0$ and by $v=\sqrt{2-a^{2}} ; \mathrm{c}=1$, then the kinetic energy of the moving object becomes negative.Perhaps this problem can be solved via Dirac-equation, where negative energies turned into charge-changing. May be this problem can be solved here, similiarly. But one can expect an „abruptly“ changing of charge by reaching this velocity-size, with may be curious again.No charge preserving by moving over this „charge-barriere“.

Third there is perhaps timetravel, so there are causality problems, because spacelike and timelike coordinates are coupled to eachother wich may cause isotropy-anomalies of spacetime.

\section{References:}


[1.] A.Einstein, über die spezielle und allgemeine Relativitätstheorie, Vieweg Braunschweig , WTB Nr. 59,21.Auflage,(1979)

[2.] A.Einstein, Grundzüge der Relativitätstheorie, Vieweg Braunschweig, WTB Nr.58,5.Auflage, (1979)

[3.] G. Feinberg,Possibility of Faster-Than-Light Particles,Phys.Rev.159,1089,(1967)

[4.] H.Hayasaka and S.Takeuchi, Anomalous weight reduction on a gyroscope's right rotations around the vertical axis on the Earth, Phys.Rev.Lett.63,2701(1989).

[5.] E.Faller,W.J.Hollander,P.G.Nelson,and M. P. Mc Hugh, Gyroscope-Weighing Experiment With A Nullresult., Physical Review Letters,64,No. 8,825,(1990)

[6.] B. Rievers and C. Lämmerzahl: High precision thermal modeling of complex systems with application to the flyby and Pioneer anomaly. Annalen der Physik, 523:439-449, 2011,

[7.]Slava G. Turyshev, Viktor T. Toth, Gary Kinsella, Siu-Chun Lee, Shing M. Lok, Jordan Ellis, Support for the thermal origin of the Pioneer anomaly Phys. Rev. Lett. 108, 241101 (2012) 
